\title{
A minimal family of sub-bases
}

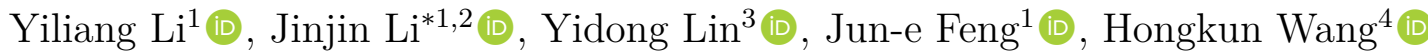 \\ ${ }^{1}$ School of Mathematics and Statistics, Minnan Normal University, Zhangzhou, Fujian 363000, P.R. \\ China \\ ${ }^{2}$ Lab of Granular Computing, Minnan Normal University, Zhangzhou, Fujian 363000, P.R. China \\ ${ }^{3}$ School of Mathematical Sciences, Xiamen University, Xiamen, Fujian 361005, P.R. China \\ ${ }^{4}$ Department of Biostatistics, Bioinformatics, and Biomathematics, Georgetown University, Washington, \\ DC 20057, USA
}

\begin{abstract}
This paper investigates a minimal family of sub-bases. First, the concept of a minimal family of sub-bases is presented and its properties are studied. Then the relationship between reducts in covering information systems and minimal families of sub-bases is discussed. Based on Boolean matrices, an approach is provided to derive a minimal family of sub-bases. Finally, experiments are conducted to illustrate the effectiveness of the proposed approach.
\end{abstract}

Mathematics Subject Classification (2010). 54A05, 54B15, 54C05, 54C10

Keywords. a minimal family of sub-bases, Boolean matrix, covering information systems, reduct

\section{Introduction}

The object of general topology is to study topological properties, which are invariants of homeomorphism [1]. Based on the properties of the topological rough membership function, Li and Zhang [6] presented the definition of sub-base reduct in a family of subbases. But there is no further research on sub-base reducts in a family of sub-bases from the point of view of general topology. In fact, sub-base reducts in a family of sub-bases are topological properties. In order to illustrate this point, this paper proposes the concept of a minimal family of sub-bases, which is equal to sub-base reducts in a family of sub-bases. In this paper, we provide a criterion of the minimal family of sub-bases. And we give an approach based on Boolean matrices to obtain the minimal family of sub-bases.

Rough set theory, introduced by Pawlak [7], provides an approach for uncertainty management. As a generalization of the classical rough set, covering rough set [21] is a useful mathematical tool to study covering information systems. A covering information system is a pair $(X, \Delta)$, where $X$ is a non-empty and finite set, and $\Delta=\left\{\mathscr{S}_{i} \mid i=1,2, \ldots, n\right\}$ is a family of coverings on $X$. According to the definition of sub-base of topological spaces, it is easy to see that a covering on $X$ is a sub-base for a topology of finite set $X$. So a family $\Delta$ of coverings is a family of sub-bases. Reducts are important problems in rough set

*Corresponding Author.

Email addresses: liyiliang1994@126.com (Y. Li), jinjinli@mnnu.edu.cn (J. Li),

yidong_lin@yeah.net (Y. Lin), fengjune@sdu.edu.cn (J. Feng), hongkun.wang@georgetown.edu (H. Wang)

Received: 01.11.2018; Accepted: 01.04.2019 
theory. In covering information systems, finding a reduct is a process to delete redundant coverings under some conditions. It is similar to deriving a minimal family of sub-bases. Then a natural question is: is there a relationship between reducts and minimal families of sub-bases? This paper shows that reducts of covering information systems and minimal families of sub-bases are equivalent.

In addition, reducts from the given information system become more and more difficult when the amount of data increases. Hence, a new research direction investigating homomorphisms or mappings between two information systems gains more attention in recent years. The motivation of study homomorphisms or mappings between information systems is to find a relatively small information system which has the same reduct as the original database [16]. Grzymala-Busse et al. [2] initially introduced the concept of homomorphism, which is used as a tool to study the relationship between information systems based on rough set. $\mathrm{Li}$ and $\mathrm{Ma}$ [5] studied some features of redundancy and reduct of complete information systems under some homomorphisms. Later, many authors [2,3,5, 9,12-20,23-28] discussed homomorphisms or mappings between information systems based on rough set. A consistent function related to coverings was proposed by Wang et al. [16]. By analyzing the consistent function related to coverings, we find that the work to structure a consistent function is a process to seek a representation element under some conditions. This process is similar to structuring a quotient space under an equivalence relation in a topological space. Hence, this paper explains consistent functions related to coverings and homomorphisms from the perspective of topology.

The remainder of this paper is organized as follows. In Section 2, the definition of a minimal family of sub-bases is presented, and its properties are investigated. Section 3 discusses the relationship between reducts and minimal families of sub-bases. Based on Boolean matrices, Section 4 proposes an approach to derive a minimal family of sub-bases. In Section 5, several numerical experiments are conducted on UCI data sets to evaluate the proposed method. Section 6 has some concluding remarks.

\section{A minimal family of sub-bases}

Suppose $\mathscr{S}_{i}$ is a sub-base for finite topological space $\left(X, \tau_{i}\right)$ for $i=1,2, \ldots, n, \Delta=$ $\left\{\mathscr{S}_{1}, \mathscr{S}_{2}, \ldots, \mathscr{S}_{n}\right\}$, and $\mathscr{S}_{\Delta}=\bigwedge_{i=1}^{n} \mathscr{S}_{i}=\left\{\bigcap_{i=1}^{n} S_{i} \mid S_{i} \in \mathscr{S}_{i}, i=1,2, \ldots, n\right\}$. Then $\mathscr{S}_{\Delta}$ is a sub-base for a topology $\tau_{\Delta}$ of finite set $X$. For each subfamily $\Delta^{\prime}$ of $\Delta$, a question is: are the topologies generated by both $\mathscr{S}_{\Delta}$ and $\mathscr{S}_{\Delta^{\prime}}$ as sub-bases the same? Now we present the definition of a minimal family of sub-bases, which keeps the topology unchanged.

Definition 2.1. Let $\mathscr{S}_{i}$ be a sub-base for finite topological space $\left(X, \tau_{i}\right)$ for $i=1,2, \ldots, n$ and $\Delta=\left\{\mathscr{S}_{1}, \mathscr{S}_{2}, \ldots, \mathscr{S}_{n}\right\}$. If the following statement holds, then the family $\Delta$ of subbases is called a minimal family of sub-bases with respect to $X$. The statement is: for any subfamily $\Delta^{\prime}$ of $\Delta$, if $\mathscr{S}_{\Delta^{\prime}}$ is a sub-base for finite topological space $\left(X, \tau_{\Delta}\right)$, then $\Delta=\Delta^{\prime}$.

In fact, two conclusions can be obtained from Definition 2.1: (1) for any family $\Delta$ of sub-bases, if there exists a subfamily $\Delta^{\prime}$ of $\Delta$ and $\Delta^{\prime} \neq \Delta$ such that $\mathscr{S}_{\Delta^{\prime}}$ is a sub-base for finite topological space $\left(X, \tau_{\Delta}\right)$, then $\Delta$ is not a minimal family of sub-bases; (2) for each subfamily $\Delta^{\prime \prime}$ of $\Delta^{\prime}$ and $\Delta^{\prime \prime} \neq \Delta^{\prime}$, if $\mathscr{S}_{\Delta^{\prime \prime}}$ is not a sub-base for finite topological space $\left(X, \tau_{\Delta}\right)$, then $\Delta^{\prime}$ is a minimal family of sub-bases. An example in [10] is employed to illustrate our idea.

Example 2.2. Suppose $\mathscr{S}_{i}$ is a sub-base for finite topological space $\left(X, \tau_{i}\right)$ for $i=1,2,3,4$ with $X=\left\{x_{1}, x_{2}, \ldots, x_{9}\right\}$. Let $\Delta=\left\{\mathscr{S}_{1}, \mathscr{S}_{2}, \mathscr{S}_{3}, \mathscr{S}_{4}\right\}$, where

$$
\begin{aligned}
& \mathscr{S}_{1}=\left\{\left\{x_{1}, x_{2}, x_{4}, x_{5}, x_{7}, x_{8}\right\},\left\{x_{2}, x_{3}, x_{5}, x_{6}, x_{8}, x_{9}\right\}\right\}, \\
& \mathscr{S}_{2}=\left\{\left\{x_{1}, x_{2}, x_{3}, x_{4}, x_{5}, x_{6}\right\},\left\{x_{4}, x_{5}, x_{6}, x_{7}, x_{8}, x_{9}\right\}\right\}, \\
& \mathscr{S}_{3}=\left\{\left\{x_{1}, x_{2}, x_{3}\right\},\left\{x_{4}, x_{5}, x_{6}, x_{7}, x_{8}, x_{9}\right\}\right\}, \\
& \mathscr{S}_{4}=\left\{\left\{x_{1}, x_{2}, x_{4}, x_{5}\right\},\left\{x_{2}, x_{3}, x_{5}, x_{6}\right\},\left\{x_{4}, x_{5}, x_{7}, x_{8}\right\},\left\{x_{5}, x_{6}, x_{8}, x_{9}\right\}\right\} .
\end{aligned}
$$


Sub-base $\mathscr{S}_{\Delta}$ is derived for finite topological space $\left(X, \tau_{\Delta}\right): \mathscr{S}_{\Delta}=\left\{\left\{x_{1}, x_{2}\right\},\left\{x_{2}\right\},\left\{x_{2}\right.\right.$, $\left.\left.x_{3}\right\},\left\{x_{4}, x_{5}\right\},\left\{x_{4}, x_{5}, x_{7}, x_{8}\right\},\left\{x_{5}\right\},\left\{x_{5}, x_{6}\right\},\left\{x_{5}, x_{6}, x_{8}, x_{9}\right\},\left\{x_{5}, x_{8}\right\}\right\}$. And it is easy to check that $\mathscr{B}_{\Delta}=\mathscr{S}_{\Delta}$, where $\mathscr{B}_{\Delta}$ is a base generated by $\mathscr{S}_{\Delta}$ as a sub-base.

Suppose $\Delta_{1}=\left\{\mathscr{S}_{3}, \mathscr{S}_{4}\right\}$. Then $\mathscr{S}_{\Delta_{1}}=\left\{\left\{x_{1}, x_{2}\right\},\left\{x_{2}, x_{3}\right\},\left\{x_{4}, x_{5}\right\},\left\{x_{5}, x_{6}\right\},\left\{x_{4}, x_{5}, x_{7}\right.\right.$, $\left.\left.x_{8}\right\},\left\{x_{5}, x_{6}, x_{8}, x_{9}\right\}\right\}$. Assume $\mathscr{B}_{\Delta_{1}}$ is a base generated by $\mathscr{S}_{\Delta_{1}}$ as a sub-base. One can examine that $\mathscr{B}_{\Delta_{1}}=\mathscr{B}_{\Delta}$, which implies that $\mathscr{S}_{\Delta_{1}}$ is a sub-base for finite topological space $\left(X, \tau_{\Delta}\right)$. So $\Delta$ is not a minimal family of sub-bases. Moreover, $\mathscr{S}_{3}$ and $\mathscr{S}_{4}$ are not sub-bases for finite topological space $\left(X, \tau_{\Delta}\right)$. Hence, $\Delta_{1}$ is a minimal family of sub-bases.

Example 2.2 shows the existence of minimal families of sub-bases, then is it unique? Reconsider Example 2.2. It is easy to find that both $\left\{\mathscr{S}_{1}, \mathscr{S}_{2}, \mathscr{S}_{3}\right\}$ and $\left\{\mathscr{S}_{3}, \mathscr{S}_{4}\right\}$ are minimal families of sub-bases. So the minimal families of sub-bases are not unique.

From [8], each point in Alexandroff spaces has a unique minimal open neighborhood. In addition, Alexandroff spaces are special topological spaces, including finite topological spaces as simple cases. So each point in finite topological spaces has a unique minimal open neighborhood. Therefore, a unique minimal open neighborhood is used to study a minimal family of sub-bases.

Suppose $\mathscr{P}$ is a family of subsets of $X$. A minimal set containing $x$ with respect to $\mathscr{P}$ is denoted by $N_{\mathscr{P}}(x)=\bigcap\{U \mid x \in U \in \mathscr{P}\}$.

Remark 2.3. Let $X$ be a finite topological space with a topology $\tau$. Suppose $\mathscr{B}$ is a base for the topological space $X$ and $\mathscr{S}$ is a sub-base for the topological space $X$. According to the definitions of base and sub-base, $N_{\tau}(x)=N_{\mathscr{B}}(x)=N_{\mathscr{S}}(x)$ for each point $x \in X$. And $N_{\tau}(x)$ is a unique minimal open neighborhood of $x$.

Theorem 2.4. Let $\mathscr{S}_{i}$ be a sub-base for finite topological space $\left(X, \tau_{i}\right)$ for $i=1,2, \ldots, n$ and $\Delta=\left\{\mathscr{S}_{1}, \mathscr{S}_{2}, \ldots, \mathscr{S}_{n}\right\}$. The family $\Delta$ of sub-bases is minimal if and only if for any subfamily $\Delta^{\prime}$ of $\Delta$, if $N_{\mathscr{S}_{\Delta^{\prime}}}(x)=N_{\mathscr{S}_{\Delta}}(x)$ for each point $x \in X$, then $\Delta=\Delta^{\prime}$.

Proof. First, we prove that for any subfamily $\Delta^{\prime}$ of $\Delta, N_{\mathscr{S}_{\Delta^{\prime}}}(x)=N_{\mathscr{S}_{\Delta}}(x)$ for each point $x \in X$ if and only if $\mathscr{S}_{\Delta^{\prime}}$ is a sub-base for finite topological space $\left(X, \tau_{\Delta}\right)$. Suppose $\mathscr{S}_{\Delta^{\prime}}$ is a sub-base for finite topological space $\left(X, \tau_{\Delta}\right)$. Then $N_{\mathscr{S}_{\Delta^{\prime}}}(x)=N_{\tau_{\Delta}}(x)=N_{\mathscr{S}_{\Delta}}(x)$ for each point $x \in X$ by Remark 2.3. Assume $\mathscr{S}_{\Delta^{\prime}}$ is a sub-base for a topology $\tau_{\Delta^{\prime}}$ of finite set $X$. Let $\mathscr{B}_{\Delta}=\left\{N_{\mathscr{S}_{\Delta}}(x) \mid x \in X\right\}$ and $\mathscr{B}_{\Delta^{\prime}}=\left\{N_{\mathscr{S}_{\Delta^{\prime}}}(x) \mid x \in X\right\}$. One can see easily that $\mathscr{B}_{\Delta}$ and $\mathscr{B}_{\Delta^{\prime}}$ are bases for finite topological spaces $\left(X, \tau_{\Delta}\right)$ and $\left(X, \tau_{\Delta^{\prime}}\right)$, respectively. Because $N_{\mathscr{S}_{\Delta}}(x)=N_{\mathscr{S}_{\Delta^{\prime}}}(x)$ for each point $x \in X$, we have $\mathscr{B}_{\Delta}=\mathscr{B}_{\Delta^{\prime}}$, which implies $\tau_{\Delta}=\tau_{\Delta^{\prime}}$. Hence, $\mathscr{S}_{\Delta^{\prime}}$ is a sub-base for finite topological space $\left(X, \tau_{\Delta}\right)$.

Suppose the family $\Delta$ of sub-bases is a minimal family of sub-bases. For any subfamily $\Delta^{\prime}$ of $\Delta$, if $N_{\mathscr{S}_{\Delta^{\prime}}}(x)=N_{\mathscr{S}_{\Delta}}(x)$ for each point $x \in X$, then $\mathscr{S}_{\Delta^{\prime}}$ is a sub-base for finite topological space $\left(X, \tau_{\Delta}\right)$. Thus, $\Delta=\Delta^{\prime}$ according to the definition of a minimal family of sub-bases. For any subfamily $\Delta^{\prime}$ of $\Delta$, suppose $\mathscr{S}_{\Delta^{\prime}}$ is a sub-base for finite topological space $\left(X, \tau_{\Delta}\right)$. From the analysis above, $N_{\mathscr{S}_{\Delta^{\prime}}}(x)=N_{\mathscr{S}_{\Delta}}(x)$ for each point $x \in X$. Then $\Delta=\Delta^{\prime}$. Therefore, the family $\Delta$ of sub-bases is the minimal one.

Theorem 2.4 shows that minimal families of sub-bases are equal to sub-base reducts in a family of sub-bases which provided by Li and Zhang [6].

Now some properties about a minimal family of sub-bases are investigated.

Theorem 2.5. Let $\mathscr{S}_{i}$ be a sub-base for finite topological space $\left(Y, \tau_{i}\right)$ for $i=1,2, \ldots, n$ and $\Delta=\left\{\mathscr{S}_{1}, \mathscr{S}_{2}, \ldots, \mathscr{S}_{n}\right\}$. Suppose $(X, \tau)$ is a finite topological space. And $f:(X, \tau) \rightarrow$ $\left(Y, \tau_{\Delta}\right)$ is continuous and onto. If $\Delta$ is a minimal family of sub-bases with respect to $Y$, then $f^{-1}(\Delta)=\left\{f^{-1}\left(\mathscr{S}_{1}\right), f^{-1}\left(\mathscr{S}_{2}\right), \ldots, f^{-1}\left(\mathscr{S}_{n}\right)\right\}$ is a minimal family of sub-bases with respect to $X$.

Proof. $\mathscr{S}_{f^{-1}(\Delta)}$ is a sub-base for a topology $\tau_{f^{-1}(\Delta)}$ of finite set $X$ because $f:(X, \tau) \rightarrow$ $\left(Y, \tau_{\Delta}\right)$ is continuous and onto. Obviously, 


$$
\begin{aligned}
f^{-1}\left(\mathscr{S}_{\Delta}\right) & =f^{-1}\left(\bigwedge_{i=1}^{n} \mathscr{S}_{i}\right) \\
& =f^{-1}\left(\left\{\bigcap_{i=1}^{n} S_{i} \mid S_{i} \in \mathscr{S}_{i}, i=1,2, \ldots, n\right\}\right) \\
& =\left\{f^{-1}\left(\bigcap_{i=1}^{n} S_{i}\right) \mid S_{i} \in \mathscr{S}_{i}, i=1,2, \ldots, n\right\} \\
& =\left\{\bigcap_{i=1}^{n} f^{-1}\left(S_{i}\right) \mid f^{-1}\left(S_{i}\right) \in f^{-1}\left(\mathscr{S}_{i}\right), i=1,2, \ldots, n\right\} \\
& =\bigwedge_{i=1}^{n} f^{-1}\left(\mathscr{S}_{i}\right)=\mathscr{S}_{f^{-1}(\Delta) .}
\end{aligned}
$$

For any subfamily $f^{-1}\left(\Delta^{\prime}\right)$ of $f^{-1}(\Delta)$, suppose $\mathscr{S}_{f^{-1}\left(\Delta^{\prime}\right)}=f^{-1}\left(\mathscr{S}_{\Delta^{\prime}}\right)$ is a sub-base for finite topological space $\left(X, \tau_{f^{-1}(\Delta)}\right)$. Then $\mathscr{S}_{\Delta^{\prime}}$ is a sub-base for finite topological space $\left(Y, \tau_{\Delta}\right)$. If not, then there exists a point $y \in Y$ such that $N_{\mathscr{S}_{\Delta^{\prime}}}(y) \neq N_{\mathscr{S}_{\Delta}}(y)$. So

$$
\begin{aligned}
N_{\mathscr{S}_{f^{-1}\left(\Delta^{\prime}\right)}}\left(f^{-1}(y)\right) & =N_{f^{-1}\left(\mathscr{S}_{\Delta^{\prime}}\right)}\left(f^{-1}(y)\right)=f^{-1}\left(N_{\mathscr{S}_{\Delta^{\prime}}}(y)\right) \\
& \neq f^{-1}\left(N_{\mathscr{S}_{\Delta}}(y)\right)=N_{f^{-1}\left(\mathscr{S}_{\Delta}\right)}\left(f^{-1}(y)\right)=N_{\mathscr{S}_{f^{-1}(\Delta)}}\left(f^{-1}(y)\right) .
\end{aligned}
$$

Thus $\mathscr{S}_{f^{-1}\left(\Delta^{\prime}\right)}$ is not a sub-base for finite topological space $\left(X, \tau_{f^{-1}(\Delta)}\right)$. It is a contradiction. Hence, $\mathscr{S}_{\Delta^{\prime}}$ is a sub-base for finite topological space $\left(Y, \tau_{\Delta}\right)$. Since $\Delta$ is a minimal family of sub-bases with respect to $Y$, by Definition $2.1, \Delta=\Delta^{\prime}$. That is $f^{-1}(\Delta)=f^{-1}\left(\Delta^{\prime}\right)$. Therefore, $f^{-1}(\Delta)$ is a minimal family of sub-bases with respect to $X$.

By Theorem 2.5, a minimal family of sub-bases remains invariant under $f^{-1}: Y \rightarrow X$ if and only if $f$ is continuous and onto. When a minimal family of sub-bases remains invariant under a mapping $f$, what conditions does the mapping $f$ satisfy? In order to answer this question, we prove a proposition first.

Definition 2.6 ([4]). Let $f: X \rightarrow Y, g: Y \rightarrow Z$ be two mappings. We call subset $\{(x, z)$ : there exists $y \in Y$ such that $f(x)=y, g(y)=z\}$ a composition of $f$ and $g$. The composition of $f$ and $g$ is denoted by $f \circ g: X \rightarrow Z$. For each $x \in X,(f \circ g)(x)=g(f(x))$.

Proposition 2.7. Let $\mathscr{S}_{i}$ be a sub-base for finite topological space $\left(X, \tau_{i}\right)$ for $i=1,2, \ldots, n$, $\Delta=\left\{\mathscr{S}_{1}, \mathscr{S}_{2}, \ldots, \mathscr{S}_{n}\right\}$, and $Y$ be a finite topological space. For two points $x_{1}, x_{2} \in X$, define $x_{1} R x_{2}$ if $N_{\mathscr{S}_{\Delta}}\left(x_{1}\right)=N_{\mathscr{S}_{\Delta}}\left(x_{2}\right)$. Define $p:\left(X, \tau_{\Delta}\right) \rightarrow\left(X / R, \tau^{\prime}\right)$ a natural quotient mapping, where $\left(X / R, \tau^{\prime}\right)$ is a natural quotient space. Suppose a mapping $g: X / R \rightarrow Y$ is a bijection. If a mapping $f: X \rightarrow Y$ satisfies $f=p \circ g$, then the following conclusions hold:

(1) For each subset $S \in \mathscr{S}_{i} \in \Delta, x_{1} \in S$ implies $x_{2} \in S$ for any points $x_{1}, x_{2} \in S$ satisfying $f\left(x_{1}\right)=f\left(x_{2}\right)$,

(2) For any two subsets $S_{i} \in \mathscr{S}_{i}$ and $S_{j} \in \mathscr{S}_{j}$ for $i, j=1,2, \ldots, n, f\left(S_{i} \cap S_{j}\right)=$ $f\left(S_{i}\right) \cap f\left(S_{j}\right)$,

(3) $f\left(\bigcap_{i=1}^{n} S_{i}\right)=\bigcap_{i=1}^{n} f\left(S_{i}\right)$,

(4) $S=f^{-1}(f(S))$ for each subset $S \in \mathscr{S}$,

(5) $\mathscr{S}=f^{-1}(f(\mathscr{S}))$ for each element $\mathscr{S} \in \Delta$.

Proof. (1) First, we prove that $f\left(x_{1}\right)=f\left(x_{2}\right)$ implies $N_{\mathscr{S}_{\Delta}}\left(x_{1}\right)=N_{\mathscr{S}_{\Delta}}\left(x_{2}\right)$ for two points $x_{1}, x_{2} \in X$. Since $f$ satisfies $f=p \circ g, f\left(x_{1}\right)=f\left(x_{2}\right)$ implies

$$
g\left(p\left(x_{1}\right)\right)=p \circ g\left(x_{1}\right)=p \circ g\left(x_{2}\right)=g\left(p\left(x_{2}\right)\right)
$$

for any two points $x_{1}, x_{2} \in X$. Thus, $p\left(x_{1}\right)=p\left(x_{2}\right)$ because $g$ is a bijection, i.e., $\left[x_{1}\right]=\left[x_{2}\right]$. So $x_{1} R x_{2}$, which means $N_{\mathscr{S}_{\Delta}}\left(x_{1}\right)=N_{\mathscr{S}_{\Delta}}\left(x_{2}\right)$. Next, for each subset $S \in \mathscr{S}_{i}, x_{1} \in S$ means $x_{1} \in N_{\mathscr{S}_{\Delta}}\left(x_{1}\right) \subset S . N_{\mathscr{S}_{\Delta}}\left(x_{1}\right)=N_{\mathscr{S}_{\Delta}}\left(x_{2}\right)$ because $f\left(x_{1}\right)=f\left(x_{2}\right)$. It means $N_{\mathscr{S}_{\Delta}}\left(x_{2}\right) \subset S$, that is $x_{2} \in S$.

(2) First, we prove that $f\left(S_{i}\right) \cap f\left(S_{j}\right)=\varnothing$ if $S_{i} \cap S_{j}=\varnothing$. Assume $f\left(S_{i}\right) \cap f\left(S_{j}\right) \neq \varnothing$. Then there exists a point $y \in Y$ such that $y \in f\left(S_{i}\right) \cap f\left(S_{j}\right)$, i.e., $y \in f\left(S_{i}\right)$ and $y \in f\left(S_{j}\right)$. So there exist two points $x_{1} \in S_{i}$ and $x_{2} \in S_{j}$ such that $f\left(x_{1}\right)=f\left(x_{2}\right)=y$. By (1), $x_{2} \in S_{i}$. Then $x_{2} \in S_{i} \cap S_{j}$. This contradicts that $f\left(S_{i}\right) \cap f\left(S_{j}\right)=\varnothing$. Next, we prove that if $S_{i} \cap S_{j} \neq \varnothing$, then $f\left(S_{i} \cap S_{j}\right)=f\left(S_{i}\right) \cap f\left(S_{j}\right)$. Obviously, $f\left(S_{i} \cap S_{j}\right) \subset f\left(S_{i}\right) \cap f\left(S_{j}\right)$ holds. For each point $y \in f\left(S_{i}\right) \cap f\left(S_{j}\right)$, there exist two points $x_{1} \in S_{i}$ and $x_{2} \in S_{j}$ such that $f\left(x_{1}\right)=f\left(x_{2}\right)=y$. By (1), $x_{2} \in S_{i}$. Then $x_{2} \in S_{i} \cap S_{j}$, which implies $y=f\left(x_{2}\right) \in f\left(S_{i} \cap S_{j}\right)$. So $f\left(S_{i}\right) \cap f\left(S_{j}\right) \subset f\left(S_{i} \cap S_{j}\right)$. That is $f\left(S_{i} \cap S_{j}\right)=f\left(S_{i}\right) \cap f\left(S_{j}\right)$. 
(3) The proof is similar to (2).

(4) It is obvious that $S \subset f^{-1}(f(S))$ holds. For each point $x \in f^{-1}(f(S)), f(x) \in f(S)$. Thus, there exists a point $x^{\prime} \in S$ such that $f(x)=f\left(x^{\prime}\right)$. By (1), $x \in S$. So $f^{-1}(f(S)) \subset S$. That is $f^{-1}(f(S))=S$.

(5) Obviously, $\mathscr{S} \subset f^{-1}(f(\mathscr{S}))$. For each subset $S \in f^{-1}(f(\mathscr{S})), f(S) \in f(\mathscr{S})$. Then there exists a subset $S^{\prime} \in \mathscr{S}$ such that $f(S)=f\left(S^{\prime}\right)$. From (4), $S=S^{\prime} \in \mathscr{S}$ must hold. Hence, $f^{-1}(f(\mathscr{S})) \subset \mathscr{S}$, that is $\mathscr{S}=f^{-1}(f(\mathscr{S}))$.

Remark 2.8. By the proof of Proposition 2.7, if both $S_{i}$ and $S_{j}$ are elements of the same sub-base, then Proposition 2.7 (2) holds. Furthermore, for each subfamily $\Delta^{\prime}$ of $\Delta$, the following statements hold:

(1) $f\left(N_{\mathscr{S}_{\Delta^{\prime}}}(x)\right)=f\left(\bigcap\left\{S \mid x \in S \in \mathscr{S}_{\Delta^{\prime}}\right\}\right)=\bigcap\left\{f(S) \mid f(x) \in f(S) \in f\left(\mathscr{S}_{\Delta^{\prime}}\right)\right\}=$ $N_{f\left(\mathscr{S}_{\Delta^{\prime}}\right)}(f(x))$ for each point $x \in X$,

(2) $N_{\mathscr{S}_{\Delta^{\prime}}}(x)=f^{-1}\left(f\left(N_{\mathscr{S}_{\Delta^{\prime}}}(x)\right)\right)$ for each point $x \in X$.

For two points $x_{1}, x_{2} \in X$, define $x_{1} R x_{2}$ if $N_{\mathscr{S}_{\Delta}}\left(x_{1}\right)=N_{\mathscr{S}_{\Delta}}\left(x_{2}\right)$. Define $p:\left(X, \tau_{\Delta}\right) \rightarrow$ $\left(X / R, \tau^{\prime}\right)$ a natural quotient mapping, where $\left(X / R, \tau^{\prime}\right)$ is a natural quotient space. Based on the natural quotient mapping, Theorem 2.9 is proved.

Theorem 2.9. Let $\mathscr{S}_{i}$ be a sub-base for finite topological space $\left(X, \tau_{i}\right)$ for $i=1,2, \ldots, n$, $\Delta=\left\{\mathscr{S}_{1}, \mathscr{S}_{2}, \ldots, \mathscr{S}_{n}\right\}$, and $(Y, \tau)$ be a finite topological space. Suppose a mapping $g: X / R \rightarrow Y$ is a bijection. If a mapping $f:\left(X, \tau_{\Delta}\right) \rightarrow(Y, \tau)$ is an open mapping satisfying $f=p \circ g$ and $\Delta$ is a minimal family of sub-bases with respect to $X$, then $f(\Delta)=\left\{f\left(\mathscr{S}_{1}\right), f\left(\mathscr{S}_{2}\right), \ldots, f\left(\mathscr{S}_{n}\right)\right\}$ is a minimal family of sub-bases with respect to $Y$.

Proof. Obviously, $\mathscr{S}_{f(\Delta)}$ is a sub-base for a topology $\tau_{f(\Delta)}$ of finite set $Y$. By Proposition $2.7(3)$

$$
\begin{aligned}
f\left(\mathscr{S}_{\Delta}\right) & =f\left(\bigwedge_{i=1}^{n} \mathscr{S}_{i}\right) \\
& =f\left(\left\{\bigcap_{i=1}^{n} S_{i} \mid S_{i} \in \mathscr{S}_{i}, i=1,2, \ldots, n\right\}\right) \\
& =\left\{f\left(\bigcap_{i=1}^{n} S_{i}\right) \mid S_{i} \in \mathscr{S}_{i}, i=1,2, \ldots, n\right\} \\
& =\left\{\bigcap_{i=1}^{n} f\left(S_{i}\right) \mid f\left(S_{i}\right) \in f\left(\mathscr{S}_{i}\right), i=1,2, \ldots, n\right\} \\
& =\bigwedge_{i=1}^{n} f\left(\mathscr{S}_{i}\right)=\mathscr{S}_{f(\Delta)} .
\end{aligned}
$$

For any subfamily $f\left(\Delta^{\prime}\right)$ of $f(\Delta)$, suppose $\mathscr{S}_{f\left(\Delta^{\prime}\right)}=f\left(\mathscr{S}_{\Delta^{\prime}}\right)$ is a sub-base for finite topological space $\left(Y, \tau_{f(\Delta)}\right)$. Then $\mathscr{S}_{\Delta^{\prime}}$ is a sub-base for finite topological space $\left(X, \tau_{\Delta}\right)$. If not, then there exists a point $x \in X$ such that $N_{\mathscr{S}_{\Delta^{\prime}}}(x) \neq N_{\mathscr{S}_{\Delta}}(x)$. From Remark 2.8, $N_{f\left(\mathscr{S}_{\Delta^{\prime}}\right)}(f(x))=f\left(N_{\mathscr{S}_{\Delta^{\prime}}}(x)\right)$ for each point $x \in X$. Then

$$
\begin{aligned}
N_{\mathscr{S}_{f\left(\Delta^{\prime}\right)}}(f(x)) & =N_{f\left(\mathscr{S}_{\Delta^{\prime}}\right)}(f(x))=f\left(N_{\mathscr{S}_{\Delta^{\prime}}}(x)\right) \\
& \neq f\left(N_{\mathscr{S}_{\Delta}}(x)\right)=N_{f\left(\mathscr{S}_{\Delta}\right)}(f(x))=N_{\mathscr{S}_{f(\Delta)}}(f(x)) .
\end{aligned}
$$

Thus, $\mathscr{S}_{f\left(\Delta^{\prime}\right)}$ is not a sub-base for finite topological space $\left(Y, \tau_{f(\Delta)}\right)$. This contradicts that $\mathscr{S}_{\Delta^{\prime}}$ is a sub-base for finite topological space $\left(X, \tau_{\Delta}\right)$. Since $\Delta$ is a minimal family of sub-bases with respect to $X$, by Definition 2.1, $\Delta=\Delta^{\prime}$. That means $f(\Delta)=f\left(\Delta^{\prime}\right)$. Hence, $f(\Delta)$ is a minimal family of sub-bases with respect to $Y$.

With no doubt, one-to-one mappings satisfy results of Proposition 2.7. So the following corollary is provided.

Corollary 2.10. Let $\mathscr{S}_{i}$ be a sub-base for finite topological space $\left(X, \tau_{i}\right)$ for $i=1,2, \ldots, n$ and $\Delta=\left\{\mathscr{S}_{1}, \mathscr{S}_{2}, \ldots, \mathscr{S}_{n}\right\}$. Suppose $(Y, \tau)$ is a finite topological space. And $f:\left(X, \tau_{\Delta}\right) \rightarrow$ $(Y, \tau)$ is open and one-to-one. If $\Delta$ is a minimal family of sub-bases with respect to $X$, then $f(\Delta)=\left\{f\left(\mathscr{S}_{1}\right), f\left(\mathscr{S}_{2}\right), \ldots, f\left(\mathscr{S}_{n}\right)\right\}$ is a minimal family of sub-bases with respect to $f(X)$.

Proof. The proof is similar to Theorem 2.9. 


\section{Relationship between minimal families of sub-bases and reducts of covering information systems}

In this section, we restrict that universes are finite. A covering information system is a pair $(X, \Delta)$, where $X$ is a non-empty and finite set, and $\Delta=\left\{\mathscr{S}_{i} \mid i=1,2, \ldots, n\right\}$ is a family of coverings on $X$. According to the definition of sub-base of topological spaces, it is easy to see that a covering on $X$ is a sub-base for a topology of finite set $X$. So a family $\Delta$ of coverings is a family of sub-bases. Moreover, the definition of reduct is introduced in covering information systems. And finding a reduct is a process to delete redundant coverings under some conditions. It is similar to deriving a minimal family of sub-bases. Then is there a relationship between reducts and minimal families of sub-bases? Before answering this question, we present the definition of reduct.

Definition $3.1([17])$. Let $(X, \Delta)$ be a covering information system. $\Delta^{\prime} \subset \Delta$ is referred to as a reduct of $\Delta$ if $\Delta^{\prime}$ satisfies $\bigcap \Delta^{\prime}=\bigcap \Delta$ and $\cap \Delta^{\prime} \neq \bigcap\left(\Delta^{\prime} \backslash\{\mathscr{S}\}\right)$ for any covering $\mathscr{S} \in \Delta^{\prime}$, where $\bigcap_{\Delta^{\prime}}=\left\{\bigcap_{\mathscr{S} \in \Delta^{\prime}} N_{\mathscr{S}}(x) \mid x \in X\right\}$ for each subfamily $\Delta^{\prime}$ of $\Delta$.

Theorem 3.2. Let $X$ be a non-empty and finite set and $\Delta$ be a family of coverings.

(1) If $(X, \Delta)$ is a covering information system and a subfamily $\Delta^{\prime}$ of $\Delta$ is a reduct of $\Delta$, then $\Delta^{\prime}$ can be viewed as a minimal family of sub-bases.

(2) If each covering $\mathscr{S}_{i} \in \Delta$ is a sub-base for topological space $\left(X, \tau_{i}\right)$ for $i=1,2, \ldots, n$ and a subfamily $\Delta^{\prime}$ of $\Delta$ is a minimal family of sub-bases, then $\Delta^{\prime}$ can be viewed as a reduct of $\Delta$.

Proof. For any subfamily $\Delta^{\prime}$ of $\Delta$, according to the definition of $N_{\mathscr{S}_{\Delta^{\prime}}}(x), \cap \Delta^{\prime}=\bigcap \Delta$ if and only if $N_{\mathscr{S}_{\Delta^{\prime}}}(x)=N_{\mathscr{S}_{\Delta}}(x)$ for each point $x \in X$.

(1) $N_{\mathscr{S}_{\Delta^{\prime}}}(x)=N_{\mathscr{S}_{\Delta}}(x)$ for each point $x \in X$ because $\Delta^{\prime}$ is a reduct of $\Delta$. It implies that $\mathscr{S}_{\Delta^{\prime}}$ is a sub-base for finite topological space $\left(X, \tau_{\Delta}\right)$. Suppose for any covering $\mathscr{S} \in \Delta^{\prime}$, $\mathscr{S}_{\Delta^{\prime} \backslash\{\mathscr{S}\}}$ is a sub-base for finite topological space $\left(X, \tau_{\Delta}\right)$. Then $N_{\mathscr{S}_{\Delta^{\prime} \backslash\{\mathscr{S}\}}}(x)=N_{\mathscr{S}_{\Delta}}(x)$ for each point $x \in X$, which implies $N_{\mathscr{S}_{\Delta^{\prime} \backslash\{\mathscr{S}\}}}(x)=N_{\mathscr{S}_{\Delta^{\prime}}}(x)$ for each point $x \in X$. Since $\Delta^{\prime}$ is reduct of $\Delta, \cap \Delta^{\prime} \neq \bigcap\left(\Delta^{\prime} \backslash\{\mathscr{S}\}\right)$ for any covering $\mathscr{S} \in \Delta^{\prime}$. In other words, there exists a point $x \in X$ such that $N_{\mathscr{S}_{\Delta^{\prime} \backslash\{\mathscr{S}\}}}(x) \neq N_{\mathscr{S}_{\Delta^{\prime}}}(x)$. It is a contradiction. Hence, $\mathscr{S}_{\Delta^{\prime} \backslash\{\mathscr{S}\}}$ is not a sub-base for finite topological space $\left(X, \tau_{\Delta}\right)$. By Definition 2.1, $\Delta^{\prime}$ is a minimal family of sub-bases.

(2) The proof is similar to (1).

In Section 2, a minimal family of sub-bases remains invariant under some certain mappings. And Theorem 3.2 states that a reduct in covering information systems and a minimal family of sub-bases are equivalent. Therefore, the reduct also remains invariant under these certain mappings when the reduct in covering information systems is viewed as a minimal family of sub-bases. Wang et al. [16] proposed the definitions of consistent mapping and homomorphism, which keep a reduct unchanged. Since there are two different mappings which keep a reduct unchanged, are these mappings the same? The following definition is about consistent mappings.

Definition 3.3 ([16]). Let $X$ and $Y$ be two universes, $f: X \rightarrow Y$ a mapping, $\mathscr{S}=$ $\left\{S_{1}, S_{2}, \ldots, S_{n}\right\}$ a covering on $X$ and $\operatorname{Cov}(\mathscr{S})=\left\{N_{\mathscr{S}}(x) \mid x \in X\right\}$. The mapping $f$ is called a consistent function with respect to $\mathscr{S}$ if for any $x, y \in X, N_{\mathscr{S}}(x)=N_{\mathscr{S}}(y)$ whenever $f(x)=f(y)$.

By Definition 3.3, the construction of consistent function with respect to $\mathscr{S}$ is to find representation elements under some conditions. This process is similar to structure a natural quotient mapping in topological spaces. For two points $x_{1}, x_{2} \in X$, define $x_{1} R x_{2}$ if $N_{\mathscr{S}}\left(x_{1}\right)=N_{\mathscr{S}}\left(x_{2}\right)$. Define $p: X \rightarrow X / R$ a natural quotient mapping. Based on the natural quotient mapping, Theorem 3.4 is proved. 
Theorem 3.4. Let $X$ and $Y$ be two finite topological spaces, $\mathscr{S}$ a covering on $X, f$ : $X \rightarrow Y$ a mapping. If $f$ is a consistent function with respect to $\mathscr{S}$, then there exists a bijection $g: X / R \rightarrow f(X)$ such that $f$ satisfies $f=p \circ g$.

Proof. Obviously, there exists a mapping $g$ such that $f=p \circ g$. Suppose $g\left(\left[x_{1}\right]\right)=g\left(\left[x_{2}\right]\right)$ for any two points $x_{1}, x_{2} \in X . p(x)=[x]$ for each point $x \in X$, because $p$ is a natural quotient mapping. Then

$$
f\left(x_{1}\right)=p \circ g\left(x_{1}\right)=g\left(p\left(x_{1}\right)\right)=g\left(\left[x_{1}\right]\right)=g\left(\left[x_{2}\right]\right)=g\left(p\left(x_{2}\right)\right)=p \circ g\left(x_{2}\right)=f\left(x_{2}\right) .
$$

Since $f$ is a consistent function with respect to $\mathscr{S}$, by Definition $3.3, N_{\mathscr{S}}\left(x_{1}\right)=N_{\mathscr{S}}\left(x_{2}\right)$. It implies $x_{1} R x_{2}$. Then $\left[x_{1}\right]=\left[x_{2}\right]$, that is $g$ is one-to-one. Because $f: X \rightarrow f(X)$ is onto and $p$ is a natural quotient mapping, $g$ is onto. So $g$ is a bijection.

According to the definition of consistent function, homomorphisms are defined in [16].

Definition $3.5([16])$. Let $(X, \Delta)$ be a covering information system. If $f$ is a consistent function with respect to each $\mathscr{S}_{i} \in \Delta$, then $f$ is referred to as a homomorphism on $(X, \Delta)$.

Wang et al. [16] proves that $f$ is a consistent function with respect to $\mathscr{S}_{1}$ and $\mathscr{S}_{2}$ if and only if $f$ is a consistent function with respect to $\mathscr{S}_{1} \cap \mathscr{S}_{2}=\left\{N_{\mathscr{S}_{1}}(x) \cap N_{\mathscr{S}_{2}}(x) \mid x \in X\right\}$. Based on mathematical induction, if $f$ is a consistent function with respect to each covering $\mathscr{S} \in \Delta$, then $f$ is a consistent function with respect to $\mathscr{S}_{\Delta}$. In other words, if $f$ is a homomorphism on $(X . \Delta)$, then $f$ is a consistent function with respect to $\mathscr{S}_{\Delta}$. So the following corollary holds according to Theorem 3.4.

Corollary 3.6. Let $X$ and $Y$ be two finite topological spaces, and $f: X \rightarrow Y$ a mapping. Suppose $\Delta=\left\{\mathscr{S}_{1}, \mathscr{S}_{2}, \ldots, \mathscr{S}_{n}\right\}$ is a family of coverings on $X$. If $f$ is a homomorphism on $(X, \Delta)$, then there exists a bijection $g: X / R \rightarrow f(X)$ such that $f$ satisfies $f=p \circ g$.

Consider $f: X \rightarrow f(X)$. By Corollary 3.6, if $f$ is a homomorphism on $(X, \Delta)$, then $f$ is the composition $p \circ g$, where $p$ is a natural quotient mapping and $g$ is a bijection. According to Theorem $3.2(1)$, a reduct $\Delta^{\prime}$ of covering information system $(X, \Delta)$ can be viewed as a minimal family of sub-bases with respect to $X$. By Theorem $2.9, f\left(\Delta^{\prime}\right)$ is a minimal family of sub-bases with respect to $f(X)$. Then $f\left(\Delta^{\prime}\right)$ can be viewed as a reduct of $f(\Delta)$ according to Theorem $3.2(2)$. The above analysis illustrates that the necessity of the following lemma, which is proposed by Wang et al in [16], is equal to Theorem 2.9 presented in this paper. On the other hand, $f: X \rightarrow f(X)$ is onto. If $f\left(\Delta^{\prime}\right)$ is a reduct of $f(\Delta)$, then $f\left(\Delta^{\prime}\right)$ can be viewed as a minimal family of sub-bases with respect to $f(X)$. By Theorem 2.5, $f^{-1}\left(f\left(\Delta^{\prime}\right)\right)$ is a minimal family of sub-bases with respect to $X$. From Proposition $2.7(5)$, one can see that $f^{-1}(f(\mathscr{S}))=\mathscr{S}$ for each sub-base $\mathscr{S} \in \Delta^{\prime}$. Then $f^{-1}\left(f\left(\Delta^{\prime}\right)\right)=\Delta^{\prime}$, which implies $\Delta^{\prime}$ is a minimal family of sub-bases with respect to $X$. Thus, $\Delta^{\prime}$ is a reduct of $\Delta$. Therefore, we conclude that the sufficiency of the following lemma and Theorem 2.5 introduced in this paper are equivalent.

Lemma $3.7([16])$. Let $(X, \Delta)$ be a covering information system, $\Delta^{\prime} \subset \Delta$. If $f$ is a homomorphism on $(X, \Delta)$, then $\Delta^{\prime}$ is a reduct of $\Delta$ if and only if $f\left(\Delta^{\prime}\right)$ is a reduct of $f(\Delta)$.

Wang et al. also defined another homomorphism in [16].

Definition $3.8([16])$. Let $(Y, \Delta)$ be a covering information system and $f: X \rightarrow Y$ be a mapping. If $f$ is surjective, then $f^{-1}$ is referred to as a homomorphism from $(Y, \Delta)$ to $\left(X, f^{-1}(\Delta)\right)$.

Based on homomorphisms from $(Y, \Delta)$ to $\left(X, f^{-1}(\Delta)\right)$, Wang et al. obtained the following lemma in [16].

Lemma $3.9([16])$. Suppose $(Y, \Delta)$ is a covering information system, $\left(X, f^{-1}(\Delta)\right)$ is an induced covering information system of $(Y, \Delta)$, and $f^{-1}$ is a homomorphism from $(Y, \Delta)$ 
to $\left(X, f^{-1}(\Delta)\right)$ and $\Delta^{\prime} \subset \Delta$. Then $\Delta^{\prime}$ is a reduct of $\Delta$ if and only if $f^{-1}\left(\Delta^{\prime}\right)$ is a reduct of $f^{-1}(\Delta)$.

We will show Lemma 3.9 is equal to Theorem 2.5 introduced in this paper. First, if $\Delta^{\prime}$ is a reduct of $\Delta$, by Theorem $3.2(1)$, then $\Delta^{\prime}$ can be viewed as a minimal family of sub-bases with respect to $Y$. Next $f$ is onto according to the definition of homomorphism from $(Y, \Delta)$ to $\left(X, f^{-1}(\Delta)\right)$. By Theorem $2.5, f^{-1}\left(\Delta^{\prime}\right)$ is a minimal family of sub-bases with respect to $X$. So $f^{-1}\left(\Delta^{\prime}\right)$ is a reduct of $f^{-1}(\Delta)$ from Theorem $3.2(2)$. In another word, the necessity of Lemma 3.9 and Theorem 2.5 are equivalent. Besides, $f^{-1}$ is also onto. If $f^{-1}\left(\Delta^{\prime}\right)$ is a reduct of $f^{-1}(\Delta)$, then $f^{-1}\left(\Delta^{\prime}\right)$ is a minimal family of sub-bases with respect to $X$. By Theorem $2.5, \Delta^{\prime}=f\left(f^{-1}\left(\Delta^{\prime}\right)\right)$ is a minimal family of sub-bases with respect to $Y$. So $\Delta^{\prime}$ is a reduct of $\Delta$. Therefore, the sufficiency of Lemma 3.9 and Theorem 2.5 are equivalent.

\section{An approach to obtain a minimal family of sub-bases}

In Section 2, a minimal family of sub-bases is presented. And a criterion of a minimal family of sub-bases is provided. In fact, it is hard to obtain a minimal family of sub-bases via this criterion. So an approach based on Boolean matrices will be given to derive a minimal family of sub-bases. First, the following definition is proposed.

Definition 4.1 ([10]). Let $X=\left\{x_{1}, x_{2}, \ldots, x_{m}\right\}$ and $A \subset X$. The characteristic function is defined as $f(A)=\left(f_{1}, f_{2}, \ldots, f_{m}\right)^{\prime}$ (' denotes the transpose throughout this paper), where

$$
f_{i}= \begin{cases}1, & x_{i} \in A \\ 0, & x_{i} \notin A\end{cases}
$$

From Definition 4.1, characteristic function $f(A)$ shows the relationship between each point of $X$ and a subset $A$ of $X$. For example, if $X=\left\{x_{1}, x_{2}, x_{3}, x_{4}, x_{5}, x_{6}\right\}$ and $A=$ $\left\{x_{2}, x_{3}, x_{6}\right\}$, then $f(A)=(0,1,1,0,0,1)^{\prime}$.

Definition 4.2. Let $\mathscr{P}$ be a family of subsets of $X$ with $X=\left\{x_{1}, x_{2}, \ldots, x_{m}\right\}$ and $\mathscr{P}=$ $\left\{P_{1}, P_{2}, \ldots, P_{k}\right\}$. The characteristic matrix of $\mathscr{P}$ is defined as $M_{\mathscr{P}}=\left(f\left(P_{1}\right), f\left(P_{2}\right), \ldots\right.$, $\left.f\left(P_{k}\right)\right)$.

Obviously, $M_{\mathscr{P}}$ is a Boolean matrix with size $m \times k$. Let $\mathscr{S}$ be a sub-base for a finite topological space $X$. A neighborhood Boolean matrix of sub-base $\mathscr{S}$ is defined.

Definition 4.3. Let $\mathscr{S}_{i}$ be a sub-base for finite topological space $\left(X, \tau_{i}\right)$ for $i=1,2, \ldots, n$ with $X=\left\{x_{1}, x_{2}, \ldots, x_{m}\right\}$, and $\Delta=\left\{\mathscr{S}_{1}, \mathscr{S}_{2}, \ldots, \mathscr{S}_{n}\right\}$. Define a neighborhood Boolean matrix of each sub-base $\mathscr{S} \in \Delta$ as $N m(\mathscr{S})=\left(f\left(N_{\mathscr{S}}\left(x_{1}\right)\right), f\left(N_{\mathscr{S}}\left(x_{2}\right)\right), \ldots, f\left(N_{\mathscr{S}}\left(x_{m}\right)\right)\right)$, and a neighborhood Boolean matrix of $\Delta$ as $N m\left(\mathscr{S}_{\Delta}\right)=\left(f\left(N_{\mathscr{S}_{\Delta}}\left(x_{1}\right)\right), f\left(N_{\mathscr{S}_{\Delta}}\left(x_{2}\right)\right), \ldots\right.$, $\left.f\left(N_{\mathscr{S}_{\Delta}}\left(x_{m}\right)\right)\right)$.

Denote the $i$-th row and $j$-th column element of neighborhood Boolean matrix $N m(\mathscr{S})$ as $N m(\mathscr{S})(i, j)$. In fact, $N m(\mathscr{S})(i, j)=1$ means $x_{i} \in N_{\mathscr{S}}\left(x_{j}\right)$ and $N m(\mathscr{S})(i, j)=0$ means $x_{i} \notin N_{\mathscr{S}}\left(x_{j}\right)$. If $x_{i} \notin N_{\mathscr{S}}\left(x_{j}\right)$ for any points $x_{i}, x_{j} \in X$, then we call that $x_{i}$ and $x_{j}$ are discernible by sub-base $\mathscr{S}$. Hence, $N m(\mathscr{S})(i, j)=0$ implies that $x_{i}$ and $x_{j}$ can be discernible by sub-base $\mathscr{S}$.

Definition $4.4([11])$. Let $M=\left(m_{i j}\right)_{n \times m}$ be a matrix. Define a matrix operator $\sim$ as $\sim M=\left(\sim m_{i j}\right)_{n \times m}$, where

$$
\sim m_{i j}= \begin{cases}1, & m_{i j}=0 \\ 0, & m_{i j} \neq 0\end{cases}
$$

Definition 4.5 ([22]). Let $A=\left(a_{i j}\right)_{n \times m}$ and $B=\left(b_{i j}\right)_{n \times m}$ be two matrices. The Hadamard product of $A$ and $B$ is defined as $A \circ B=\left(a_{i j} b_{i j}\right)_{n \times m}$. 
Two operators introduced above will be used to calculate neighborhood Boolean matrices.

Theorem 4.6. Let $\mathscr{S}_{i}$ be a sub-base for finite topological space $\left(X, \tau_{i}\right)$ for $i=1,2, \ldots, n$ and $\Delta=\left\{\mathscr{S}_{1}, \mathscr{S}_{2}, \ldots, \mathscr{S}_{n}\right\}$. Then the following statements hold:

(1) $N m(\mathscr{S})=\sim\left(\left(\sim M_{\mathscr{S}}\right) M_{\mathscr{S}}^{\prime}\right)$ for each sub-base $\mathscr{S} \in \Delta$,

(2) $N m\left(\mathscr{S}_{\Delta}\right)=N m\left(\mathscr{S}_{1}\right) \circ N m\left(\mathscr{S}_{2}\right) \circ \cdots \circ N m\left(\mathscr{S}_{n}\right)$.

Proof. For each sub-base $\mathscr{S} \in \Delta$, suppose $\mathscr{S}=\left\{S_{1}, S_{2}, \ldots, S_{k}\right\}$. And the $i$-th element of column vector $f(S)$ is denoted by $f(S)(i)$. For each matrix $M$, denote its $j$-th column vector as $M(\cdot, j)$, and its $i$-th row and $j$-th column element as $M(i, j)$.

(1) Suppose $x_{i} \in N_{\mathscr{S}}\left(x_{j}\right)$. It means $N m(\mathscr{S})(i, j)=1$. Next we prove that $(\sim((\sim$ $\left.\left.\left.M_{\mathscr{S}}\right) M_{\mathscr{S}}^{\prime}\right)\right)(i, j)=1$. It is obvious that

$$
\left(\left(\sim M_{\mathscr{S}}\right) M_{\mathscr{S}}^{\prime}\right)(\cdot, j)=\sum_{1 \leq l \leq k} M_{\mathscr{S}}^{\prime}(l, j)\left(\sim f\left(S_{l}\right)\right) .
$$

According to Definition $4.2, M_{\mathscr{S}}^{\prime}(l, j)=1$ means $x_{j} \in S_{l}$, and $M_{\mathscr{S}}^{\prime}(l, j)=0$ implies $x_{j} \notin S_{l}$. Thus,

$$
\left(\left(\sim M_{\mathscr{S}}\right) M_{\mathscr{S}}^{\prime}\right)(\cdot, j)=\sum_{1 \leq l \leq k} M_{\mathscr{S}}^{\prime}(l, j)\left(\sim f\left(S_{l}\right)\right)=\sum_{x_{j} \in S_{l}}\left(\sim f\left(S_{l}\right)\right) .
$$

For each point $x_{i} \in X, x_{i} \in N_{\mathscr{S}}\left(x_{j}\right)$ means $x_{i} \in S_{l}$ for each subset $S_{l}$ satisfying $x_{j} \in S_{l}$. This implies $f\left(S_{l}\right)(i)=1$ and $\left(\sim f\left(S_{l}\right)\right)(i)=0$ for each subset $S_{l}$ satisfying $x_{j} \in S_{l}$. Hence, for each point $x_{i} \in N_{\mathscr{S}}\left(x_{j}\right)$,

$$
\left(\sum_{x_{j} \in S_{l}}\left(\sim f\left(S_{l}\right)\right)\right)(i)=\sum_{x_{j} \in S_{l}}\left(\left(\sim f\left(S_{l}\right)\right)(i)\right)=0 .
$$

That is $\sim\left(\sum_{x_{j} \in S_{l}}\left(\sim f\left(S_{l}\right)\right)\right)(i)=1$. Therefore, $\left(\sim\left(\left(\sim M_{\mathscr{S}}\right) M_{\mathscr{S}}^{\prime}\right)\right)(i, j)=1$. Assume $x_{i} \notin N_{\mathscr{S}}\left(x_{j}\right)$. Then we have $\operatorname{Nm}(\mathscr{S})(i, j)=0$. It is similar to proving that $(\sim((\sim$ $\left.\left.M_{\mathscr{S}}\right) M_{\mathscr{S}}^{\prime}\right)(i, j)=0$. So it is easy to conclude that

$$
\operatorname{Nm}(\mathscr{S})=\sim\left(\left(\sim M_{\mathscr{S}}\right) M_{\mathscr{S}}^{\prime}\right) .
$$

(2) If $N m\left(\mathscr{S}_{\Delta}\right)(i, j)=1$, then $x_{i} \in N_{\mathscr{S}_{\Delta}}\left(x_{j}\right)$. According to the definitions of $\mathscr{S}_{\Delta}$ and minimal open neighborhood of each point $x \in X$, we get $x_{i} \in N_{\mathscr{S}}\left(x_{j}\right)$ for each element $\mathscr{S} \in \Delta$, which implies $N m(\mathscr{S})(i, j)=1$ for each element $\mathscr{S} \in \Delta$. So

$$
\left(N m\left(\mathscr{S}_{1}\right) \circ N m\left(\mathscr{S}_{2}\right) \circ \cdots \circ N m\left(\mathscr{S}_{n}\right)\right)(i, j)=1 \text {. }
$$

If $N m\left(\mathscr{S}_{\Delta}\right)(i, j)=0$, then it is similar to proving that

$$
\left(N m\left(\mathscr{S}_{1}\right) \circ N m\left(\mathscr{S}_{2}\right) \circ \cdots \circ N m\left(\mathscr{S}_{n}\right)\right)(i, j)=0 \text {. }
$$

Consequently, $\operatorname{Nm}\left(\mathscr{S}_{\Delta}\right)=N m\left(\mathscr{S}_{1}\right) \circ N m\left(\mathscr{S}_{2}\right) \circ \cdots \circ N m\left(\mathscr{S}_{n}\right)$.

Theorem 4.6 shows that the discernible power of $\mathscr{S}_{\Delta}$ is larger than $\mathscr{S}_{\Delta^{\prime}}$ for each subfamily $\Delta^{\prime}$ of $\Delta$. Or the discernible power of $\Delta$ is larger than each subfamily $\Delta^{\prime}$ of $\Delta$. The following example in [17] is given to calculate neighborhood Boolean matrices by Theorem 4.6 .

Example 4.7. Suppose $\mathscr{S}_{i}$ is a sub-base for finite topological space $\left(X, \tau_{i}\right)$ for $i=1,2,3,4$ with $X=\left\{x_{1}, x_{2}, \ldots, x_{15}\right\}$.Let $\Delta=\left\{\mathscr{S}_{1}, \mathscr{S}_{2}, \mathscr{S}_{3}, \mathscr{S}_{4}\right\}$, where

$$
\begin{aligned}
& \mathscr{S}_{1}=\left\{\left\{x_{1}, x_{2}, x_{3}, x_{4}, x_{5}, x_{8}, x_{10}, x_{15}\right\},\left\{x_{3}, x_{5}, x_{7}, x_{11}, x_{12}\right\},\left\{x_{4}, x_{6}, x_{8}, x_{9}, x_{10}, x_{13}, x_{14}\right\}\right\}, \\
& \mathscr{S}_{2}=\left\{\left\{x_{1}, x_{2}, x_{3}, x_{4}, x_{5}, x_{7}, x_{8}, x_{10}, x_{11}, x_{12}, x_{15}\right\},\left\{x_{3}, x_{4}, x_{5}, x_{8}, x_{10}\right\},\left\{x_{3}, x_{5}, x_{6}, x_{9},\right.\right. \\
&\left.\left.x_{13}, x_{14}\right\}\right\}, \\
& \mathscr{S}_{3}=\left\{\left\{x_{1}, x_{2}, x_{3}, x_{4}, x_{5}, x_{8}, x_{10}, x_{15}\right\},\left\{x_{6}, x_{9}, x_{13}, x_{14}\right\},\left\{x_{4}, x_{7}, x_{8}, x_{10}, x_{11}, x_{12}\right\}\right\}, \\
& \mathscr{S}_{4}=\left\{\left\{x_{1}, x_{2}, x_{3}, x_{5}, x_{15}\right\},\left\{x_{6}, x_{7}, x_{9}, x_{11}, x_{12}, x_{13}, x_{14}\right\},\left\{x_{3}, x_{4}, x_{5}, x_{8}, x_{10}\right\}\right\} .
\end{aligned}
$$

The characteristic matrices of $\mathscr{S}_{i}$ for $i=1,2,3,4$ are obtained: 


$$
M_{\mathscr{S}_{1}}=\left(\begin{array}{ccc}
1 & 0 & 0 \\
1 & 0 & 0 \\
1 & 1 & 0 \\
1 & 0 & 1 \\
1 & 1 & 0 \\
0 & 0 & 1 \\
0 & 1 & 0 \\
1 & 0 & 1 \\
0 & 0 & 1 \\
1 & 0 & 1 \\
0 & 1 & 0 \\
0 & 1 & 0 \\
0 & 0 & 1 \\
0 & 0 & 1 \\
1 & 0 & 0
\end{array}\right), M_{\mathscr{S}_{2}}=\left(\begin{array}{ccc}
1 & 0 & 0 \\
1 & 0 & 0 \\
1 & 1 & 1 \\
1 & 1 & 0 \\
1 & 1 & 1 \\
0 & 0 & 1 \\
1 & 0 & 0 \\
1 & 1 & 0 \\
0 & 0 & 1 \\
1 & 1 & 0 \\
1 & 0 & 0 \\
1 & 0 & 0 \\
0 & 0 & 1 \\
0 & 0 & 1 \\
1 & 0 & 0
\end{array}\right), M_{\mathscr{S}_{3}}=\left(\begin{array}{ccc}
1 & 0 & 0 \\
1 & 0 & 0 \\
1 & 0 & 0 \\
1 & 0 & 1 \\
1 & 0 & 0 \\
0 & 1 & 0 \\
0 & 0 & 1 \\
1 & 0 & 1 \\
0 & 1 & 0 \\
1 & 0 & 1 \\
0 & 0 & 1 \\
0 & 0 & 1 \\
0 & 1 & 0 \\
0 & 1 & 0 \\
1 & 0 & 0
\end{array}\right), M_{\mathscr{S}_{4}}=\left(\begin{array}{lll}
1 & 0 & 0 \\
1 & 0 & 0 \\
1 & 0 & 1 \\
0 & 0 & 1 \\
1 & 0 & 1 \\
0 & 1 & 0 \\
0 & 1 & 0 \\
0 & 0 & 1 \\
0 & 1 & 0 \\
0 & 0 & 1 \\
0 & 1 & 0 \\
0 & 1 & 0 \\
0 & 1 & 0 \\
0 & 1 & 0 \\
1 & 0 & 0
\end{array}\right) .
$$

According to Theorem 4.6, neighborhood Boolean matrices are derived.

$$
\begin{aligned}
& N m\left(\mathscr{S}_{1}\right)=\left(\begin{array}{lllllllllllllll}
1 & 1 & 0 & 0 & 0 & 0 & 0 & 0 & 0 & 0 & 0 & 0 & 0 & 0 & 1 \\
1 & 1 & 0 & 0 & 0 & 0 & 0 & 0 & 0 & 0 & 0 & 0 & 0 & 0 & 1 \\
1 & 1 & 1 & 0 & 1 & 0 & 1 & 0 & 0 & 0 & 1 & 1 & 0 & 0 & 1 \\
1 & 1 & 0 & 1 & 0 & 1 & 0 & 1 & 1 & 1 & 0 & 0 & 1 & 1 & 1 \\
1 & 1 & 1 & 0 & 1 & 0 & 1 & 0 & 0 & 0 & 1 & 1 & 0 & 0 & 1 \\
0 & 0 & 0 & 0 & 0 & 1 & 0 & 0 & 1 & 0 & 0 & 0 & 1 & 1 & 0 \\
0 & 0 & 0 & 0 & 0 & 0 & 1 & 0 & 0 & 0 & 1 & 1 & 0 & 0 & 0 \\
1 & 1 & 0 & 1 & 0 & 1 & 0 & 1 & 1 & 1 & 0 & 0 & 1 & 1 & 1 \\
0 & 0 & 0 & 0 & 0 & 1 & 0 & 0 & 1 & 0 & 0 & 0 & 1 & 1 & 0 \\
1 & 1 & 0 & 1 & 0 & 1 & 0 & 1 & 1 & 1 & 0 & 0 & 1 & 1 & 1 \\
0 & 0 & 0 & 0 & 0 & 0 & 1 & 0 & 0 & 0 & 1 & 1 & 0 & 0 & 0 \\
0 & 0 & 0 & 0 & 0 & 0 & 1 & 0 & 0 & 0 & 1 & 1 & 0 & 0 & 0 \\
0 & 0 & 0 & 0 & 0 & 1 & 0 & 0 & 1 & 0 & 0 & 0 & 1 & 1 & 0 \\
0 & 0 & 0 & 0 & 0 & 1 & 0 & 0 & 1 & 0 & 0 & 0 & 1 & 1 & 0 \\
1 & 1 & 0 & 0 & 0 & 0 & 0 & 0 & 0 & 0 & 0 & 0 & 0 & 0 & 1
\end{array}\right), \\
& N m\left(\mathscr{S}_{2}\right)=\left(\begin{array}{lllllllllllllll}
1 & 1 & 0 & 0 & 0 & 0 & 1 & 0 & 0 & 0 & 1 & 1 & 0 & 0 & 1 \\
1 & 1 & 0 & 0 & 0 & 0 & 1 & 0 & 0 & 0 & 1 & 1 & 0 & 0 & 1 \\
1 & 1 & 1 & 1 & 1 & 1 & 1 & 1 & 1 & 1 & 1 & 1 & 1 & 1 & 1 \\
1 & 1 & 0 & 1 & 0 & 0 & 1 & 1 & 0 & 1 & 1 & 1 & 0 & 0 & 1 \\
1 & 1 & 1 & 1 & 1 & 1 & 1 & 1 & 1 & 1 & 1 & 1 & 1 & 1 & 1 \\
0 & 0 & 0 & 0 & 0 & 1 & 0 & 0 & 1 & 0 & 0 & 0 & 1 & 1 & 0 \\
1 & 1 & 0 & 0 & 0 & 0 & 1 & 0 & 0 & 0 & 1 & 1 & 0 & 0 & 1 \\
1 & 1 & 0 & 1 & 0 & 0 & 1 & 1 & 0 & 1 & 1 & 1 & 0 & 0 & 1 \\
0 & 0 & 0 & 0 & 0 & 1 & 0 & 0 & 1 & 0 & 0 & 0 & 1 & 1 & 0 \\
1 & 1 & 0 & 1 & 0 & 0 & 1 & 1 & 0 & 1 & 1 & 1 & 0 & 0 & 1 \\
1 & 1 & 0 & 0 & 0 & 0 & 1 & 0 & 0 & 0 & 1 & 1 & 0 & 0 & 1 \\
1 & 1 & 0 & 0 & 0 & 0 & 1 & 0 & 0 & 0 & 1 & 1 & 0 & 0 & 1 \\
0 & 0 & 0 & 0 & 0 & 1 & 0 & 0 & 1 & 0 & 0 & 0 & 1 & 1 & 0 \\
0 & 0 & 0 & 0 & 0 & 1 & 0 & 0 & 1 & 0 & 0 & 0 & 1 & 1 & 0 \\
1 & 1 & 0 & 0 & 0 & 0 & 1 & 0 & 0 & 0 & 1 & 1 & 0 & 0 & 1
\end{array}\right), \\
& N m\left(\mathscr{S}_{3}\right)=\left(\begin{array}{lllllllllllllll}
1 & 1 & 1 & 0 & 1 & 0 & 0 & 0 & 0 & 0 & 0 & 0 & 0 & 0 & 1 \\
1 & 1 & 1 & 0 & 1 & 0 & 0 & 0 & 0 & 0 & 0 & 0 & 0 & 0 & 1 \\
1 & 1 & 1 & 0 & 1 & 0 & 0 & 0 & 0 & 0 & 0 & 0 & 0 & 0 & 1 \\
1 & 1 & 1 & 1 & 1 & 0 & 1 & 1 & 0 & 1 & 1 & 1 & 0 & 0 & 1 \\
1 & 1 & 1 & 0 & 1 & 0 & 0 & 0 & 0 & 0 & 0 & 0 & 0 & 0 & 1 \\
0 & 0 & 0 & 0 & 0 & 1 & 0 & 0 & 1 & 0 & 0 & 0 & 1 & 1 & 0 \\
0 & 0 & 0 & 0 & 0 & 0 & 1 & 0 & 0 & 0 & 1 & 1 & 0 & 0 & 0 \\
1 & 1 & 1 & 1 & 1 & 0 & 1 & 1 & 0 & 1 & 1 & 1 & 0 & 0 & 1 \\
0 & 0 & 0 & 0 & 0 & 1 & 0 & 0 & 1 & 0 & 0 & 0 & 1 & 1 & 0 \\
1 & 1 & 1 & 1 & 1 & 0 & 1 & 1 & 0 & 1 & 1 & 1 & 0 & 0 & 1 \\
0 & 0 & 0 & 0 & 0 & 0 & 1 & 0 & 0 & 0 & 1 & 1 & 0 & 0 & 0 \\
0 & 0 & 0 & 0 & 0 & 0 & 1 & 0 & 0 & 0 & 1 & 1 & 0 & 0 & 0 \\
0 & 0 & 0 & 0 & 0 & 1 & 0 & 0 & 1 & 0 & 0 & 0 & 1 & 1 & 0 \\
0 & 0 & 0 & 0 & 0 & 1 & 0 & 0 & 1 & 0 & 0 & 0 & 1 & 1 & 0 \\
1 & 1 & 1 & 0 & 1 & 0 & 0 & 0 & 0 & 0 & 0 & 0 & 0 & 0 & 1
\end{array}\right), \\
& N m\left(\mathscr{S}_{4}\right)=\left(\begin{array}{lllllllllllllll}
1 & 1 & 0 & 0 & 0 & 0 & 0 & 0 & 0 & 0 & 0 & 0 & 0 & 0 & 1 \\
1 & 1 & 0 & 0 & 0 & 0 & 0 & 0 & 0 & 0 & 0 & 0 & 0 & 0 & 1 \\
1 & 1 & 1 & 1 & 1 & 0 & 0 & 1 & 0 & 1 & 0 & 0 & 0 & 0 & 1 \\
0 & 0 & 0 & 1 & 0 & 0 & 0 & 1 & 0 & 1 & 0 & 0 & 0 & 0 & 0 \\
1 & 1 & 1 & 1 & 1 & 0 & 0 & 1 & 0 & 1 & 0 & 0 & 0 & 0 & 1 \\
0 & 0 & 0 & 0 & 0 & 1 & 1 & 0 & 1 & 0 & 1 & 1 & 1 & 1 & 0 \\
0 & 0 & 0 & 0 & 0 & 1 & 1 & 0 & 1 & 0 & 1 & 1 & 1 & 1 & 0 \\
0 & 0 & 0 & 1 & 0 & 0 & 0 & 1 & 0 & 1 & 0 & 0 & 0 & 0 & 0 \\
0 & 0 & 0 & 0 & 0 & 1 & 1 & 0 & 1 & 0 & 1 & 1 & 1 & 1 & 0 \\
0 & 0 & 0 & 1 & 0 & 0 & 0 & 1 & 0 & 1 & 0 & 0 & 0 & 0 & 0 \\
0 & 0 & 0 & 0 & 0 & 1 & 1 & 0 & 1 & 0 & 1 & 1 & 1 & 1 & 0 \\
0 & 0 & 0 & 0 & 0 & 1 & 1 & 0 & 1 & 0 & 1 & 1 & 1 & 1 & 0 \\
0 & 0 & 0 & 0 & 0 & 1 & 1 & 0 & 1 & 0 & 1 & 1 & 1 & 1 & 0 \\
0 & 0 & 0 & 0 & 0 & 1 & 1 & 0 & 1 & 0 & 1 & 1 & 1 & 1 & 0 \\
1 & 1 & 0 & 0 & 0 & 0 & 0 & 0 & 0 & 0 & 0 & 0 & 0 & 0 & 1
\end{array}\right) .
\end{aligned}
$$


Thus,

$$
N m\left(\mathscr{S}_{\Delta}\right)=\left(\begin{array}{lllllllllllllll}
1 & 1 & 0 & 0 & 0 & 0 & 0 & 0 & 0 & 0 & 0 & 0 & 0 & 0 & 1 \\
1 & 1 & 0 & 0 & 0 & 0 & 0 & 0 & 0 & 0 & 0 & 0 & 0 & 0 & 1 \\
1 & 1 & 1 & 0 & 1 & 0 & 0 & 0 & 0 & 0 & 0 & 0 & 0 & 0 & 1 \\
0 & 0 & 0 & 1 & 0 & 0 & 0 & 1 & 0 & 1 & 0 & 0 & 0 & 0 & 0 \\
1 & 1 & 1 & 0 & 1 & 0 & 0 & 0 & 0 & 0 & 0 & 0 & 0 & 0 & 1 \\
0 & 0 & 0 & 0 & 0 & 1 & 0 & 0 & 1 & 0 & 0 & 0 & 1 & 1 & 0 \\
0 & 0 & 0 & 0 & 0 & 0 & 1 & 0 & 0 & 0 & 1 & 1 & 0 & 0 & 0 \\
0 & 0 & 0 & 1 & 0 & 0 & 0 & 1 & 0 & 1 & 0 & 0 & 0 & 0 & 0 \\
0 & 0 & 0 & 0 & 0 & 1 & 0 & 0 & 1 & 0 & 0 & 0 & 1 & 1 & 0 \\
0 & 0 & 0 & 1 & 0 & 0 & 0 & 1 & 0 & 1 & 0 & 0 & 0 & 0 & 0 \\
0 & 0 & 0 & 0 & 0 & 0 & 1 & 0 & 0 & 0 & 1 & 1 & 0 & 0 & 0 \\
0 & 0 & 0 & 0 & 0 & 0 & 1 & 0 & 0 & 0 & 1 & 1 & 0 & 0 & 0 \\
0 & 0 & 0 & 0 & 0 & 1 & 0 & 0 & 1 & 0 & 0 & 0 & 1 & 1 & 0 \\
0 & 0 & 0 & 0 & 0 & 1 & 0 & 0 & 1 & 0 & 0 & 0 & 1 & 1 & 0 \\
1 & 1 & 0 & 0 & 0 & 0 & 0 & 0 & 0 & 0 & 0 & 0 & 0 & 0 & 1
\end{array}\right) .
$$

From Example 2.2, we find that $\mathscr{S}_{3}$ is the common element of minimal families of subbases $\left\{\mathscr{S}_{1}, \mathscr{S}_{2}, \mathscr{S}_{3}\right\}$ and $\left\{\mathscr{S}_{3}, \mathscr{S}_{4}\right\}$. Then $\mathscr{S}_{3}$ is more important than others for a minimal family of sub-bases. A natural question is: is there an element $\mathscr{S} \in \Delta$ such that $\mathscr{S}$ belongs to all the minimal families of sub-bases? If there is, then what properties does the element have? The concept of core is proposed in the following definition.

Definition 4.8. Let $\mathscr{S}_{i}$ be a sub-base for finite topological space $\left(X, \tau_{i}\right)$ for $i=1,2, \ldots, n$ and $\Delta=\left\{\mathscr{S}_{1}, \mathscr{S}_{2}, \ldots, \mathscr{S}_{n}\right\}$. The element $\mathscr{S}$ is called a core if $\mathscr{S}$ is an element of all the minimal families of sub-bases.

The characteristics of a minimal family of sub-bases and core can be described via neighborhood Boolean matrices.

Theorem 4.9. Let $\mathscr{S}_{i}$ be a sub-base for finite topological space $\left(X, \tau_{i}\right)$ for $i=1,2, \ldots, n$, and $\Delta=\left\{\mathscr{S}_{1}, \mathscr{S}_{2}, \ldots, \mathscr{S}_{n}\right\}$. Then the following results hold:

(1) For each subfamily $\Delta^{\prime}$ of $\Delta, \mathscr{S}_{\Delta^{\prime}}$ is a sub-base for finite topological space $\left(X, \tau_{\Delta}\right)$ if and only if $N m\left(\mathscr{S}_{\Delta}\right)=N m\left(\mathscr{S}_{\Delta^{\prime}}\right)$;

(2) $\mathscr{S}$ is a core if and only if $N m\left(\mathscr{S}_{\Delta \backslash\{\mathscr{S}\}}\right) \neq N m\left(\mathscr{S}_{\Delta}\right)$ for each element $\mathscr{S} \in \Delta$.

Proof. (1) From the proof of Theorem 2.4, for each subfamily $\Delta^{\prime}$ of $\Delta, \mathscr{S}_{\Delta^{\prime}}$ is a sub-base for finite topological space $\left(X, \tau_{\Delta}\right)$ if and only if $N_{\mathscr{S}_{\Delta}}(x)=N_{\mathscr{S}_{\Delta^{\prime}}}(x)$ for each point $x \in X$. By the definition of neighborhood Boolean matrix, it is equal to $N m\left(\mathscr{S}_{\Delta}\right)=N m\left(\mathscr{S}_{\Delta^{\prime}}\right)$.

(2) Assume $N m\left(\mathscr{S}_{\Delta \backslash\{\mathscr{S}\}}\right)=N m\left(\mathscr{S}_{\Delta}\right)$. From (1), $\mathscr{S}_{\Delta \backslash\{\mathscr{S}\}}$ is a sub-base for finite topological space $\left(X, \tau_{\Delta}\right)$. Obviously, there exists a subfamily $\Delta^{\prime}$ of $\Delta \backslash\{\mathscr{S}\}$ such that $\Delta^{\prime}$ is a minimal family of sub-bases, and $\mathscr{S} \notin \Delta^{\prime}$. Because $\mathscr{S}$ is a core, $\mathscr{S}$ is an element of all the minimal families of sub-bases. It is a contradiction. Hence, $N m\left(\mathscr{S}_{\Delta \backslash\{\mathscr{S}\}}\right) \neq N m\left(\mathscr{S}_{\Delta}\right)$.

For each matrix $M$, denote its $i$-th row and $j$-th column element as $M(i, j)$. Suppose a subfamily $\Delta^{\prime}$ of $\Delta \backslash\{\mathscr{S}\}$ is a minimal family of sub-bases. From (1), $N m\left(\mathscr{S}_{\Delta}\right)=$ $N m\left(\mathscr{S}_{\Delta^{\prime}}\right)$. Because $N m\left(\mathscr{S}_{\Delta}\right) \neq N m\left(\mathscr{S}_{\Delta \backslash\{\mathscr{S}\}}\right)$, there exist $N m\left(\mathscr{S}_{\Delta}\right)(i, j)$ and $N m\left(\mathscr{S}_{\Delta \backslash\{\mathscr{S}\}}\right)$ $(i, j)$ such that $N m\left(\mathscr{S}_{\Delta}\right)(i, j)=0$ but $N m\left(\mathscr{S}_{\Delta \backslash \mathscr{S}}\right)(i, j)=1$. That is $N m\left(\mathscr{S}_{\Delta^{\prime}}\right)(i, j)=0$ but $N m\left(\mathscr{S}_{\Delta \backslash\{\mathscr{S}\}}\right)(i, j)=1$. By Theorem 4.6, $N m\left(\mathscr{S}_{\Delta \backslash\{\mathscr{S}\}}\right)(i, j)=1$ means $N m\left(\mathscr{S}^{\prime}\right)(i, j)$ $=1$ for each element $\mathscr{S}^{\prime} \in \Delta \backslash\{\mathscr{S}\}$. It is contradictory to $N m\left(\mathscr{S}_{\Delta^{\prime}}\right)(i, j)=0$. So $\Delta^{\prime}$ is not a minimal family of sub-bases. Therefore, $\mathscr{S}$ is a core.

Corollary 4.10. Let $\mathscr{S}_{i}$ be a sub-base for finite topological space $\left(X, \tau_{i}\right)$ for $i=1,2, \ldots, n$ and $\Delta=\left\{\mathscr{S}_{1}, \mathscr{S}_{2}, \ldots, \mathscr{S}_{n}\right\}$. Then a subfamily $\Delta^{\prime}$ of $\Delta$ is a minimal family of sub-bases if and only if $\Delta^{\prime}$ is a minimal subfamily of $\Delta$ satisfying $N m\left(\mathscr{S}_{\Delta}\right)=N m\left(\mathscr{S}_{\Delta^{\prime}}\right)$.

Based on the results above, a heuristic algorithm is presented to find a minimal family of sub-bases.

Steps 3-5 are to compute all the cores and their time complexity is not more than $O\left(\sum_{\mathscr{S} \in \Delta}|X|^{2}|\mathscr{S}|\right)$. A sub-base with the maximal discernible power is added into a minimal family $\Delta^{\prime}$ of sub-bases in step 9 , whose time complexity is $O\left(\sum_{i=1}^{|\Delta|-1}|X|^{2}(|\Delta|-i)\right)$. So the time complexity of Algorithm 1 is $O\left(\sum_{\mathscr{S} \in \Delta}|X|^{2}|\mathscr{S}|+\sum_{i=0}^{|\Delta|-1}|X|^{2}(|\Delta|-i)\right)$. 


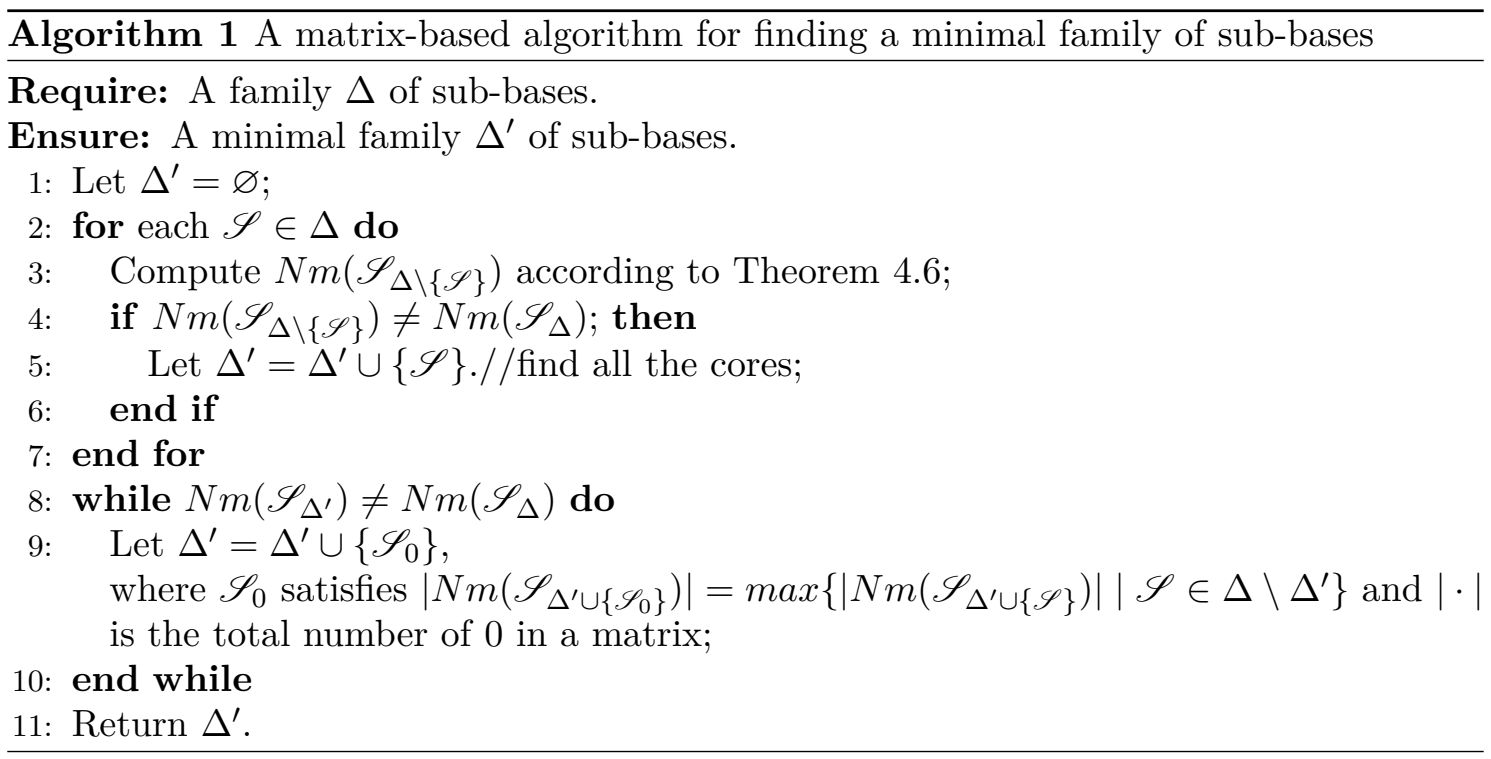

The following example uses Algorithm 1 to compute a minimal family of sub-bases.

Example 4.11. Re-discuss Example 4.7. According to Algorithm 1, we can obtain that: $N m\left(\mathscr{S}_{\Delta \backslash\left\{\mathscr{S}_{1}\right\}}\right)=N m\left(\mathscr{S}_{\Delta}\right), N m\left(\mathscr{S}_{\Delta \backslash\left\{\mathscr{S}_{2}\right\}}\right)=N m\left(\mathscr{S}_{\Delta}\right), N m\left(\mathscr{S}_{\Delta \backslash\left\{\mathscr{S}_{3}\right\}}\right)=N m\left(\mathscr{S}_{\Delta}\right)$, $N m\left(\mathscr{S}_{\Delta \backslash\left\{\mathscr{S}_{4}\right\}}\right) \neq N m\left(\mathscr{S}_{\Delta}\right)$. So $\mathscr{S}_{4}$ is a core. In addition, $\left|N m\left(\mathscr{S}_{\left\{\mathscr{S}_{1}, \mathscr{S}_{4}\right\}}\right)\right|=\max$ $\left\{\left|N m\left(\mathscr{S}_{\left\{\mathscr{S}_{4}\right\} \cup\{\mathscr{S}\}}\right)\right| \mid \mathscr{S} \in \Delta \backslash\left\{\mathscr{S}_{4}\right\}\right\}$ and $N m\left(\mathscr{S}_{\Delta_{1}}\right)=N m\left(\mathscr{S}_{\Delta}\right)$. Hence, $\Delta_{1}=\left\{\mathscr{S}_{1}, \mathscr{S}_{4}\right\}$ is a minimal family of sub-bases on $X$.

\section{Numerical experiments}

To further illustrate the effectiveness of the proposed algorithm, we choose 18 data sets from UCI to conduct several numerical experiments. These data sets are described in Table 1. Decision attributes in data sets are removed, and the rest of these data are normalized. In order to obtain an original family of sub-bases from data sets, a parameter $\varepsilon$ is used to control the size of the neighborhood of each object in each data set. For each attribute in each data set, each object has a neighborhood. And the family of neighborhoods of all objects is regarded as a covering. Since a covering can be viewed as a sub-base for a topological space, the family of neighborhoods of all objects will be a sub-base for a topological space. Hence, given an $\varepsilon$, a family of sub-bases is generated by all attributes in each data set. In our numerical experiments, we take $\varepsilon=0.4$ to generate a family of sub-bases with respect to each data set.

The experiments are performed on a personal computer with Windows 10 and an Inter (R) Core (TM) i7-6700 CPU @ $3.40 \mathrm{GHz} 3.41 \mathrm{GHz}$ with 8 GB of memory. The algorithms are implemented using Matlab R2017a.

Table 1. Data set description

\begin{tabular}{ccccccccc}
\hline Data sets & Sample & Attributes & Data sets & Sample & Attributes & Data sets & Sample & Attributes \\
\hline breast & 84 & 9216 & crx & 690 & 15 & derm & 366 & 34 \\
diabe & 768 & 8 & gearboxA & 1603 & 72 & gearboxB & 1603 & 72 \\
gearboxC & 1603 & 72 & gearboxD & 1603 & 72 & gene1 & 84 & 9216 \\
heart & 270 & 13 & hepatitis & 155 & 19 & horse & 368 & 22 \\
sonar & 208 & 60 & wdbc & 569 & 30 & wine & 178 & 13 \\
wpbc & 198 & 33 & yale & 165 & 1024 & zoo & 101 & 16 \\
\hline
\end{tabular}


As seen in Table 2, sub-bases' number in a minimal family of sub-bases is dramatically reduced. It shows that Algorithm 1 is effective in deleting redundant elements and providing a minimal family of sub-bases.

Table 2. Sub-bases' numbers in a minimal family of sub-bases

\begin{tabular}{ccc}
\hline Date sets & Sub-bases' numbers in a original family of sub-bases & Sub-bases' numbers in a minimal family of sub-bases \\
\hline breast & 9216 & 2 \\
crx & 15 & 12 \\
derm & 34 & 2 \\
diabe & 8 & 8 \\
gearboxA & 72 & 5 \\
gearboxB & 72 & 7 \\
gearboxC & 72 & 7 \\
gearboxD & 72 & 6 \\
gene1 & 9216 & 2 \\
heart & 13 & 1 \\
hepatitis & 19 & 1 \\
horse & 22 & 13 \\
sonar & 60 & 9 \\
wdbc & 30 & 18 \\
wine & 13 & 7 \\
wpbc & 33 & 7 \\
yale & 1024 & 5 \\
zoo & 16 & 1 \\
\hline
\end{tabular}

Time consumption on finding a minimal family of sub-bases is shown in Table 3 . It can be observed that Algorithm 1 does not cost much time on all the data sets. It further turns out that Algorithm 1 is feasible in finding a minimal family of sub-bases. In addition, the effect of objects' number on time consumption is larger than that of sub-bases' numbers. For example, for data set breast with 84 objects and 9216 sub-bases, the time for finding a minimal family of sub-bases is 113.76s. But for data set gearboxD with 1603 objects and 72 sub-bases, the time for finding a minimal family of sub-bases is $365.01 \mathrm{~s}$. Between the two data sets, the gap of sub-bases' number is far greater than that of objects' number. However, time consumption of data set breast with more sub-bases is less than data set gearboxD with more objects. The reason for it is as follows, that sub-bases' numbers do not affect objects' number, but objects' number affects subsets' number in each sub-base, which affects the computing speed of neighborhood Boolean matrix.

Table 3. Time consumptions on finding a minimal family of sub-bases

\begin{tabular}{cccccc}
\hline Data sets & Times(s) & Data sets & Times(s) & Data sets & Times(s) \\
\hline breast & 113.76 & crx & 2.48 & derm & 0.94 \\
diabe & 2.66 & gearboxA & 330.69 & gearboxB & 354.69 \\
gearboxC & 335.53 & gearboxD & 365.01 & gene1 & 122.23 \\
heart & 0.50 & hepatitis & 0.31 & horse & 2.35 \\
sonar & 3.91 & wdbc & 12.68 & wine & 0.60 \\
wpbc & 2.03 & yale & 48.29 & zoo & 0.07 \\
\hline
\end{tabular}

\section{Conclusion}

In this paper, the definition of a minimal family of sub-bases has been presented. Moreover, a criterion of a minimal family of sub-bases has been provided. According to this criterion, an approach based on Boolean matrices has been proposed to obtain a minimal family of sub-bases. In order to illustrate the effectiveness of the obtained approach, we have conducted several numerical experiments on UCI data sets. Although the presented approach is feasible in finding a minimal family of sub-bases, there is no way to show that the derived result is optimal. Moreover, the relationship between reducts in covering information systems and minimal families of sub-bases has been discussed. 
Acknowledgment. This work is supported by National Natural Science Foundation of China (Nos. 11871259, 61379021, 11701258, 61773371) and Natural Science Foundation of Fujian Province (Nos. 2019J01748). The authors thank the anonymous reviewers for their constructive comments.

\section{References}

[1] R. Engelking, General Topology, Heldermann Verlag, Berlin, 1989.

[2] J.M. Graymala-Busse, Algebraic properties of knowledge representation systems, in: Proceedings of the ACM SIGART international symposium on Methodologies for intelligent systems, ACM, 432-440, 1986.

[3] G.M. Lang, Q.G. Li and L.K. Guo, Homomorphisms-based attribute reduction of dynamic fuzzy covering information systems, Int. J. Gen. Syst. 44 (7-8), 791-811, 2015.

[4] J.J. Li, K.D. Li and S. Lin, Introduction of Basic Topology, Science Press, Beijing, 2009 (in Chinese).

[5] D.Y. Li and Y.C. Ma, Invariant characters of information systems under some homomorphisms, Inf. Sci. 129 (1-4), 211-220, 2000.

[6] J.J. Li and Y.L. Zhang, Reduction of subbases and its applications, Utilitas Math. 82 (3), 179-192, 2010.

[7] Z. Pawlak, Rough sets, Int. J. Comput. Inf. Sci. 11 (5), 341-356, 1982.

[8] T. Speer, A short study of Alexandroff spaces, at http://arxiv.org/abs/0708.2136, 2007.

[9] A.H. Tan, J.J. Li and G.P. Lin, Extended results on the relationship between information systems, Inf. Sci. 290, 156-173, 2015.

[10] A.H. Tan, J.J. Li and G.P. Lin and Y.J. Lin, Fast approach to knowledge acquistion in covering information systems using matrix operations, Knowl.-Based Syst. 79, 90-98, 2015.

[11] A.H. Tan, J.J. Li, Y.J. Lin and G.P. Lin, Matrix-based set approximations and reductions in covering decision information systems, Int. J. Approx. Reason. 59, 68-80, 2015.

[12] E.C.C. Tsang, C.Z. Wang, D.G. Chen, C.X. Wu and Q.H. Hu, Communication between information systems using fuzzy rough sets, IEEE Trans. Fuzzy syst. 21 (3), 527-540, 2013.

[13] C.Z. Wang, W.Y. Bao, X.X. Wang and Q. He, A mapping between fuzzy covering information systems, Proc. 2013 IEEE International Conference on Granular Computing, 315-319.

[14] C.Z. Wang, D.G. Chen and Q.H. Hu, Some invariant properties of ordered information systems under homomorphism, Sci. China: Inf. Sci. 53 (9), 1816-1825, 2010.

[15] C.Z. Wang, D.G. Chen and Q.H. Hu, Fuzzy information systems and their homomorphisms, Fuzzy Sets Syst. 249, 128-138, 2014.

[16] C.Z. Wang, D.G. Chen, B.Q. Sun and Q.H. Hu, Communication between information systems with covering based rough sets, Inf. Sci. 216, 17-33, 2012.

[17] C.Z. Wang, D.G. Chen, C. Wu and Q.H. Hu, Data compression with homomorphism in covering information systems, Int. J. Approx. Reason. 52 (4), 519-525, 2011.

[18] C.Z. Wang, D.G. Chen and L.K. Zhu, Homomorphisms between fuzzy information systems, Appl. Math. Lett. 22 (7), 1045-1050, 2009.

[19] C.Z. Wang, C.X. Wu, D.G. Chen and W.J. Du, Some properties of relation information systems under homomorphisms, Appl. Math. Lett. 21 (9), 940-945, 2008.

[20] C.Z. Wang, C.X. Wu, D.G. Chen, Q.H. Hu and C. Wu, Communicating between information systems, Inf. Sci. 178 (16), 3228-3239, 2008. 
[21] W. Żakowski, Approximations in the space $(U, \Pi)$, Demonstr. Math. 16 (3), 761-769, 1983.

[22] X.D. Zhang, Matrix analysis and applications, Tsinghua University Press, Beijing, 2004 (in Chinese).

[23] P. Zhu, Covering rough sets based on neighborhoods: An approach without using neighborhoods, Int. J. Approx. Reason. 52 (3), 461-472, 2011.

[24] P. Zhu and Q.Y. Wen, Some improved results on communication between information systems, Inf. Sci. 180 (18), 3521-3531, 2010.

[25] P. Zhu and Q.Y. Wen, Homomorphisms between fuzzy information systems revisited, Appl. Math. Lett. 24 (9), 1548-1553, 2011.

[26] P. Zhu and Q.Y. Wen, A note on communicating between information systems based on including degrees, Int. J. Gen. Syst. 40 (8), 837-840, 2011.

[27] P. Zhu, H.Y. Xie and Q.Y. Wen, A unified definition of consistent functions, Fund. Inf. 135 (3), 331-340, 2014.

[28] P. Zhu, H.Y. Xie and Q.Y. Wen, A unified view of consistent functions, Soft Comput. 21 (9), 2189-2199, 2017. 